\title{
Zum Ausmaß der bundespolitischen Parteipolitikverflechtung bei Landtagswahlen in Deutschland 1996 bis 2000
}

\author{
Ivar Krumpal und Heiko Rauhut*
}

Die empirische Wahlforschung in Deutschland hat in der jüngsten Zeit großes Interesse an der engen Verflechtung zwischen Bundes- und Landespolitik gezeigt. Eine zentrale Fragestellung hierbei lautet, ob es sich bei Landtagswahlen primär um Bundestestwahlen handelt oder ob vielmehr landes(partei)politische Aspekte die individuellen Wahlpräferenzen beeinflussen. Während einige Studien die Relevanz der übergeordneten bundespolitischen Verhältnisse bei der Erklärung von Landtagswahlergebnissen betonen ${ }^{1}$, vermuten andere Forschungsarbeiten eine zunehmende Entkoppelung der Landtagswahlen vom bundespolitischen Geschehen. Insbesondere Daniel Hough und Charlie Jeffery argumentieren, dass die Wähler bei den Landtagswahlen zunehmend nach regionalen Gesichtspunkten wählten und der Einfluss der Bundesparteien abgenommen habe. Ihrer Regionalisierungsthese zufolge würden sich die Wähler bei Landtagswahlen stärker an den Landesparteien orientieren und ihre Wahlpräferenzen weniger von den Bundesparteien abhängig machen. ${ }^{2}$ Diese These soll hier einem empirischen Test mit Individualdaten unterzogen werden. Anhand eines mehrstufigen statistischen Analyserahmens wird das Ausmaß der bundespolitischen Durchdringung von Landtagswahlen quantifiziert. Die Untersuchung folgt dabei insbesondere der Logik der Wiederholung: Um widerstreitende Thesen über den relativen Einfluss von bundes- und landespolitischen Faktoren zu testen, wird zunächst ein einfaches Modell formuliert. Dieses wird dann wiederholt auf identische Art und Weise in mehreren Datensätzen geschätzt. Anschließend werden die Ergebnisse einzelner Bundesländer miteinander verglichen.

Die Stärke des Einflusses der Bundespolitik auf das Abstimmungsverhalten bei Landtagswahlen war auch das zentrale Thema in Heft 3/2007 der Zeitschrift für Parlaments-

Heiko Rauhut arbeitet jetzt an der ETH Zürich, der Beitrag stammt allerdings noch aus seiner Tätigkeit an der Universität Leipzig.

1 Reiner Dinkel, Der Zusammenhang zwischen Bundes- und Landtagswahlergebnissen, in: PVS, 18. Jg. (1977), S. 348 - 359; ders., Zur Gesetzmäßigkeit der Trendverschiebungen zwischen Landtags- und Bundestagswahlen, in: ZParl, 12. Jg. (1981), H. 1, S. 135 - 139; ders., Landtagswahlen unter dem Einfluss der Bundespolitik: Die Erfahrung der letzten Legislaturperioden, in: Jürgen W. Falter / Hans Rattinger / Klaus G. Troitzsch (Hrsg.), Wahlen und politische Einstellungen in der Bundesrepublik Deutschland, Frankfurt am Main 1989, S. 253 - 262. Frank Decker und Julia von Blumenthal schreiben hierzu: „Landtagswahlen haben ... immer mehr den Charakter von ,Zwischen- beziehungsweise Testwahlen' angenommen und fungieren als Stimmungsbarometer für die Bundespolitik." Frank Decker / Julia von Blumenthal, Die bundespolitische Durchdringung der Landtagswahlen. Eine empirische Analyse von 1970 bis 2001, in: ZParl, 33. Jg. (2002), H. 1, S. 144 - 165, S. 145.

2 „Wir vermuten auf Landesebene eine eigenständige territoriale Dynamik im Wahlverhalten, die zur nationalen Wahldynamik ein zunehmend loses Verhältnis einnimmt." Daniel Hough / Charlie Jeffery, Landtagswahlen: Bundestestwahlen oder Regionalwahlen?, in: ZParl, 34. Jg. (2003), H. 1, S. $79-94$, S. 81. 
fragen. ${ }^{3}$ Auf inhaltlicher Ebene ergänzt der vorliegende Beitrag diese Studien, legt allerdings den Analysefokus auf den direkten Vergleich von Wählerwahrnehmungen zwischen alten und neuen Bundesländern. Auf forschungsmethodischer Ebene wird in Übereinstimmung mit Dieter Ohr und Markus Klein argumentiert, dass für einen solchen Vergleich identisch spezifizierte Schätzmodelle in Form eines replikativen Surveys besonders geeignet sind. ${ }^{4}$

\section{Landtagswahlen im Lichte einer allgemeinen Theorie der Nebenwahlen}

Um Wahlverhalten in subnationalen Kontexten theoretisch zu fundieren, entwickelten Karlheinz Reif und Hermann Schmitt eine allgemeine Theorie der „Nebenwahlen“" . Anlass für die Entwicklung dieses Analyserahmens waren die ersten direkten Wahlen zum Europäischen Parlament 1979. Dabei wurde eine wichtige Unterscheidung zwischen zwei verschiedenen Typen von Wahlen eingeführt:

(1) Als „Hauptwahlen“ werden Wahlen eingestuft, bei denen über die Machtverhältnisse auf nationaler Ebene abgestimmt wird. ${ }^{6}$ Bei parlamentarischen Regierungssystemen wie etwa Deutschland oder Großbritannien fallen unter diese Kategorie die Wahlen zum nationalen Parlament, bei präsidentiellen Systemen wie den USA bezieht sich dieser Terminus auf die Präsidentschaftswahlen. Dabei wird diese Kategorie von Wahlen als erstrangig („first-order elections“) bezeichnet, denn hier wird entschieden, wer zukünftig im Land das Sagen hat.

(2) „Nebenwahlen“ werden im Gegensatz dazu in der subjektiven Wählerwahrnehmung als weniger wichtig eingeschätzt. Sie werden als zweitrangig („second-order elections“) bezeichnet. Neben den Wahlen zum Europäischen Parlament fallen in diese Kategorie alle subnationalen Wahlen (Kommunalwahlen, Regionalwahlen, Landtagswahlen). Im Falle von präsidentiellen Regierungssystemen gehören hierzu auch die Wahlen von Repräsentanten der legislativen Staatsorgane.

3 Oscar W. Gabriel / Everhard Holtmann, Ober sticht Unter? Zum Einfluss der Bundespolitik auf Landtagswahlen: Kontext, theoretischer Rahmen und Analysemodelle, in: ZParl, 38. Jg. (2007), H. 3, S. $445-462$.

4 Dieter Ohr / Markus Klein, Landtagswahlen in Nordrhein-Westfalen 1990 - 2005: keine Dominanz der Bundespolitik, in: ZParl, 38. Jg. (2007), H. 3, S. 541 - 549. Durch die Wiederholung ein und desselben statistischen Modells in mehreren Datensätzen lassen sich bekannte Thesen einem besonders strengen Robustheitstest unterziehen. Das methodische Vorgehen stützt sich dabei in erster Linie auf die wissenschaftstheoretische Leitidee der Replikation und kumulativer Wissensvermehrung: „Have we identified an empirical regularity that has some degree of invariance? (...) Generally replication and prediction of new results provide a harsher and more useful validation regime than statistical testing of many models on one data set." David A. Freedman, Statistical Models and Shoe Leather, in: Sociological Methodology, 21. Jg. (1991), S. 291 - 313, S. 306.

5 Karlheinz Reif/ Hermann Schmitt, Nine Second-Order National Elections: A Conceptual Framework for the Analysis of European Election Results, in: European Journal of Political Research, 8. Jg. (1980), S. 3 - 44; Karlheinz Reif, Nationale Regierungsparteien verlieren die Wahl zum Europäischen Parlament 1984, in: ZParl, 15. Jg. (1984), H. 3, S. 341 - 352; ders. I Hermann Schmitt / Pippa Norris, Second-order Elections, in: European Journal of Political Research, Bd. 31 (1997), S. 109 - 124.

6 Diese wird auch als „Hauptarena“ bezeichnet. Vgl. Karlheinz Reif, a.a.O. (1984), S. 344. Wahlen, die in subnationalen beziehungsweise europäischen Kontexten stattfinden, werden unter dem Begriff „Nebenarena“ subsumiert. 
Der zentrale Aspekt von Nebenwahlen besteht darin, dass weniger auf dem Spiel steht („less is at stake“). Dies hat für Wahlen, welche in einer Nebenarena des jeweiligen politischen Systems stattfinden, folgende wichtigen Implikationen ${ }^{7}$ :

(1) Bei zweitrangigen Wahlen ist regelmäßig eine niedrigere Wahlbeteiligung zu beobachten als bei Nationalwahlen. Darin kommt zum Ausdruck, dass die Wähler diesem Wahltypus geringere Relevanz beimessen.

(2) Die Bürger experimentieren eher mit kleinen oder neuen Parteien. Während es bei Hauptwahlen um die Besetzung der wichtigsten Spitzenpositionen im politischen System geht, stehen bei Nebenwahlen weniger wichtige Ämter zur Disposition. Bei Wahlen zum nationalen Parlament möchten viele Wähler ihre Stimme nicht „verschwenden“ und entscheiden sich bei ihrer Stimmabgabe für eine der großen etablierten Parteien. Bei Nebenwahlen neigen sie dagegen eher dazu, eine kleine Partei zu wählen, von der sie sich unter Umständen besser vertreten fühlen.

(3) Der höhere Anteil an ungültigen Stimmzetteln wird häufig als allgemeine Unzufriedenheit mit den Leistungen der Parteien und Spitzenpolitiker in der Hauptarena interpretiert.

(4) Die Parteien, die auf nationaler Ebene die Regierung bilden, schneiden bei Nebenwahlen in der Regel schlecht ab. Die Oppositionsparteien können dagegen häufig Stimmengewinne für sich verbuchen. Die Gründe hierfür können in einem Sanktionswahlverhalten, das sich gegen die Regierung der Hauptarena richtet, gesehen werden.

Wendet man diese Logik auf die Landtagswahlen in Deutschland an, lässt sich folgende These aufstellen: Das Abschneiden der Bundesregierungsparteien bei Landtagswahlen hängt entscheidend von der jeweils aktuellen bundespolitischen Situation ab. Oder allgemeiner formuliert: „Die Ergebnisse von Nebenwahlen entsprechen dem typischen Muster der Zustimmungskurve in der Bevölkerung für die Regierung und die sie tragenden Parteien in der Hauptarena im Verlauf des Hauptwahlzyklus. "8 Da in der Regel die Popularitätswerte der Bundesregierung im Laufe ihrer Wahlperiode stark absinken, ist auch ein entsprechend hoher Stimmenverlust bei den Landtagswahlen zu erwarten. Die empirischen Befunde von Simone Burkhart deuten in diesem Zusammenhang auf eine starke bundespolitische Durchdringung von Landtagswahlen hin. ${ }^{9}$ In ihrer empirischen Untersuchung mit Aggregatdaten kommt Burkhart zu dem Schluss, dass das Ausmaß der Stimmenverluste der Bundesregierungsparteien bei den Landtagswahlen von der Höhe ihres Popularitätsverlustes seit Amtsantritt abhängt. Neuere empirische Untersuchungen für die Bundesrepublik haben Susanne Lohmann und andere, Brian J. Gaines und Christophe Crombez vorgelegt. ${ }^{10}$ Die jüngsten empirischen Studien zum Landtagswahlverhalten stellen ebenso einen Bundeseinfluss auf das Abstimmungsverhalten bei Landtagswahlen fest, wobei die Stärke dieses Einflusses in Abhängigkeit von der gewählten statistischen Modellspezifikation variiert. ${ }^{11}$

7 Karlheinz Reif/ Hermann Schmitt, a.a.O., S. 9 f.

8 Karlheinz Reif, a.a.O. (1984), S. 344.

9 Simone Burkhart, Parteipolitikverflechtung. Über den Einfluss der Bundespolitik auf Landtagswahlentscheidungen von 1976 bis 2002, in: PVS, 46. Jg. (2005), S. $14-38$.

10 Susanne Lohmann / David W. Brady / Douglas Rivers, Party Identification, Retrospective Voting, and Moderating Elections in a Federal System: West Germany, 1961 - 1989, in: Comparative Political Studies, 30. Jg. (1997), S. 420 - 449; Brian J. Gaines / Christophe Crombez, Another Look at Connections Across German Elections, in: Journal of Theoretical Politics, 16. Jg. (2004), S. $289-319$.

11 Vgl. Oscar W. Gabriel / Everhard Holtmann, a.a.O., S. 459. 


\section{Landtagswablergebnisse: Reflektoren des bundespolitischen Klimas oder Spiegelbilder regionaler Entwicklungen?}

Die Relevanz der Bundespolitik für Landtagswahlen hat zweifelsohne Grenzen. So ist auch nach der relativen Wichtigkeit von landesspezifischen und regionalen Faktoren zu fragen. Die regelmäßig in der Zeitschrift für Parlamentsfragen veröffentlichten Landtagswahlanalysen zeichnen häufig ein recht uneinheitliches Bild. ${ }^{12}$ Dabei werden je nach allgemeiner Stimmungslage regionale Aspekte oder bundespolitische Ereignisse stärker in den Vordergrund gerückt. Es scheint schwierig, vom jeweiligen spezifischen Kontext einer konkreten Landtagswahl losgelöst eine allgemeine Aussage zu formulieren: „Der eigentümliche Charakter des bundesdeutschen Verbundföderalismus führt dazu, dass eine genaue Abgrenzung von bundes- und landespolitischer Sphäre nur schwer möglich ist. "13 Ebenso resümiert Georg Fabritius: „So stehen die Landtagswahlen und die Landespolitik nicht ganz im Schatten der Bundespolitik, sondern eher im Halbschatten. "14 Sicher steht bei Landtagswahlen weniger auf dem Spiel als bei Bundestagswahlen; indes treffen Landesregierungen auch wichtige Entscheidungen und haben somit einen entscheidenden Anteil daran, die Weichen für die zukünftige Entwicklung des jeweiligen Bundeslandes zu stellen. Zudem hängt die parteipolitische Komposition des Bundesrates direkt von den Landtagswahlergebnissen ab. ${ }^{15}$ Die Länderkammer ist im Prozess der Gesetzgebung mit beträchtlicher Vetomacht ausgestattet. Bei einer Vorherrschaft der Oppositionsparteien im Bundesrat droht der Bundesregierung insbesondere bei ihren wichtigen (zustimmungsbedürftigen) Gesetzen häufig deren Verhinderung. ${ }^{16}$ Sowohl für Politikwissenschaftler als auch für Wahlkampfstrategen stellt sich nun die interessante Frage, mit welcher Gewichtung die regionale relativ zur bundesparteipolitischen Dimension in die Landtagswahlpräferenzen der Bürger einfließt.

In der Zeit vor der deutschen Wiedervereinigung bestand weitgehender Konsens in der Fachliteratur, dass die übergeordnete bundespolitische Konstellation Landtagswahlergebnis-

12 Beispiele für Wahlstudien, die das Verhältnis von Bundes- und Landtagswahlen in Deutschland thematisieren, sind unter anderem: Peter Haungs / Eckhard Jesse, Die rheinland-pfälzische Landtagswahl vom 18. März 1979 - Anfang vom Ende der CDU-Mehrheit?, in: ZParl, 11. Jg. (1980), H. 2, S. 153 - 166; dies., Die rheinland-pfälzische Landtagswahl vom 6. März 1983: Erste „Doppelwahl“ in der Geschichte der Bundesrepublik, in: ZParl, 14. Jg. (1983), H. 4, S. 517 - 531; Wilhelm P. Bürklin / Gerhard Franz / Rüdiger Schmitt, Die hessische Landtagswahl vom 25. September 1983: Politische Neuordnung nach der „Wende“?, in: ZParl, 15. Jg. (1984), H. 2, S. 237 - 253; Rainer-Olaf Schultze, Die bayerische Landtagswahl vom 12. Oktober 1986: Stabile Verhältnisse wie nachhaltige Veränderungen, in: ZParl, 18. Jg. (1987), H. 1, S. 38 - 56; Werner Billing, Die rheinland-pfälzische Landtagswahl vom 21. April 1991: Machtwechsel in Mainz nach 44 Jahren, in: ZParl, 22. Jg. (1991), H. 4, S. 584 - 601; Klaus von Beyme, Zusammenlegung von Wahlterminen: Entlastung der Wähler - Entlastung der Politiker?, in: ZParl, 23. Jg. (1992), H. 2, S. 339 - 353; Rüdiger Schmitt-Beck, Die hessische Landtagswahl vom 19. Februar 1995: Bestätigung der Regierungsfähigkeit von Grünen, in: ZParl, 27. Jg. (1996), H. 2, S. 243 - 256.

13 Frank Decker / Julia von Blumenthal, a.a.O., S. 146.

14 Georg Fabritius, Landtagswahlen im Schatten der Bundespolitik - Auch in den Parteien eine personelle und programmatische Politikverflechtung, in: Der Bürger im Staat, 29. Jg. (1979), H. 1, S. $29-33$.

15 Vgl. Gerhard Lehmbruch, Parteienwettbewerb im Bundesstaat - Regelsysteme und Spannungslagen im politischen System der Bundesrepublik Deutschland, 3. Auflage, Wiesbaden 2000.

16 Vgl. Thomas König / Thomas Bräuninger, Wie wichtig sind die Länder für die Politik der Bundesregierung bei Einspruchs- und Zustimmungsgesetzen?, in: ZParl, 28. Jg. (1997), H. 4, S. 605 - 628. 
se deutlich beeinflusst. Insbesondere Reiner Dinkel und Georg Fabritius stellten immer wieder fest, dass das nationale politische Klima für den Ausgang einer Landtagswahl von entscheidender Bedeutung ist. ${ }^{17}$ Dieses Verhältnis, wonach Landtagswahlen vorwiegend „Bundestestwahlen“, „Pseudo-Plebiszite“ über die Bundespolitik oder gar „Bundesratswahlen" 18 seien, wurde im Rahmen der jüngeren Fachdebatte insbesondere von Daniel Hough und Charlie Jeffery in Frage gestellt: „Landtagswahlen haben seit 1990 zunehmend ihre eigene (beziehungsweise 16 eigene) Dynamik(en), werden weniger von bundespolitischen Fragen ,durchdrungen' und spiegeln eher die Landesbesonderheiten eines heterogener gewordenen Deutschlands wider. "19 Die Autoren begründen dies vor allem mit dem Aufkommen von neuen territorialen Konfliktlinien („cleavages“), die nach der Wiedervereinigung zwischen den alten und den neuen Bundesländern entstanden sind. Dagegen seien die klassischen Hauptkonfliktlinien der territorial homogeneren alten Bundesrepublik, also Bildung, Klasse und Schicht, Postmaterialismus etc. zunehmend in den Hintergrund getreten. ${ }^{20}$ Die zentrale Hauptkonfliktlinie sei jetzt im Zuge des Vereinigungsprozesses in den Gegensätzen zwischen den neuen und den alten Bundesländern zu sehen: „Diese neue Territorialität der Landtagswahlen nach der deutschen Vereinigung hat den herkömmlichen zyklischen Charakter des Verhältnisses von Landtags- zu Bundestagswahlen gesprengt. Landtagswahlen sind nicht mehr eindeutig zweitrangige Wahlen. Sie werden stattdessen zunehmend nach landeseigenen, territorialen Dynamiken entschieden. "21 Zudem gibt auch die nach 1990 veränderte Parteienlandschaft Anlass, das Verhältnis zwischen Bundespolitik und Landtagswahlen neu zu überdenken. Vor allem das Fortbestehen der PDS, die im Osten die drittstärkste politische Kraft darstellt, im Westen jedoch erst seit Fusion mit der WASG in jüngster Zeit mehr Anhänger findet, sei als Ausdruck von unterschiedlichen materiellen Interessen und Wertesystemen zu deuten, die letztendlich zu den beobachteten Unterschieden im Wahlverhalten zwischen West und Ost führen. ${ }^{22}$

Ein weiterer Aspekt ist die zunehmende Personalisierung der Wahlkämpfe, eine Tendenz, die auch bei aktuellen Landtagswahlkämpfen auszumachen ist. Bereits Dinkel konnte in seinen Untersuchungen zum bundesdeutschen Wahlzyklus feststellen, dass berühmte Landesväter einen Amtsbonus genießen und es immer wieder schaffen, dem Popularitätstrend ihrer Partei auf Bundesebene zu trotzen. ${ }^{23}$ Ein populärer Ministerpräsident kann, wenn er zum Zeitpunkt der Landtagswahl einer der Parteien der amtierenden Regierungskoalition auf Bundesebene angehört, deren Midterm-Verluste beträchtlich abmildern. Auch

17 Reiner Dinkel, a.a.O. (1977); Georg Fabritius, Wechselwirkungen zwischen Landtagswahlen und Bundespolitik, Meisenheim am Glan 1978.

18 Bereits zu Zeiten Konrad Adenauers war bei Landtagswahlkämpfen vom Bundesrat die Rede, wie folgender Wahlslogan bei der hessischen Wahlkampagne der CDU aus dem Jahre 1954 belegt: „Deine Wahl im Hessenstaat zählt im Bonner Bundesrat. Regierung Zinn stützt Ollenhauer, wählt CDU für Adenauer“, zitiert in: Georg Fabritius, a.a.O. (1979), S. 30.

19 Daniel Hough / Charlie Jeffery, a.a.O. (2003), S. 81; vgl. auch dies., The Electoral Cycle and Multi-Level Voting in Germany, in: German Politics, 10. Jg. (2001), S. 73 - 98.

20 Vgl. Rainer Schnell / Ulrich Kohler, Empirische Untersuchung einer Individualisierungshypothese am Beispiel der Parteipräferenz von 1953 - 1992, in: Kölner Zeitschrift für Soziologie und Sozialpsychologie, 47. Jg. (1995), S. 634 - 658; Daniel Hough / Charlie Jeffery, a.a.O. (2003), S. 88.

21 Daniel Hough / Charlie Jeffery, a.a.O. (2003), S. 90.

22 Ebenda, S. 89.

23 Reiner Dinkel, a.a.O. (1977). 
findet im Rahmen von Landtagswahlkämpfen oft eine bewusste Abgrenzung zur eigenen Bundespartei statt. Spezifische regionale Themen werden stattdessen in den Vordergrund gerückt: „Wir konstatieren kein einfaches Sanktionswahlverhalten, sondern ein ,Mehr-Ebenen-Wahlverhalten', wobei die Wähler unterschiedliche Wahlentscheidungen mit unterschiedlichen Begründungen für die Bundesebene verglichen mit der Landesebene treffen. "24 Wähler können, so die These, bei ihren Wahlentscheidungen relativ trennscharf zwischen Bundes- und Landesparteien unterscheiden. Diese Annahme wird im Folgenden empirisch getestet. ${ }^{25}$ Neben einer Betrachtung von aggregierten Wahlstatistiken ${ }^{26}$ werden insbesondere die zugrunde liegenden Wahlpräferenzen direkt mit individuellen Umfragedaten überprüft. Die zu testende Regionalisierungsthese lautet: Die subjektive Beurteilung der Landesparteien beeinflusst die Landtagswahlpräferenzen von Wählern stärker als deren subjektive Beurteilung der Bundesparteien.

\section{Empirischer Test der Parteipolitikverflechtung bei Landtagswahlen}

\subsection{Datenbasis}

Um die Regionalisierungshypothese empirisch zu testen, werden alle Landtagswahlstudien der Forschungsgruppe Wahlen aus dem Zeitraum von 1996 bis 2000 herangezogen. Dies sind insgesamt 17 Individualdatensätze, wobei 15 Bundesländer einmal, Schleswig-Holstein zweimal (1996 und 2000) vertreten ist. Die befragten Zielpersonen wurden zunächst zufällig ermittelt. Anschließend wurden die Daten anhand standardisierter Fragebögen im Rahmen von Telefoninterviews erhoben. Eine genaue Beschreibung der Datenbasis befindet sich im Anhang.

24 Daniel Hough / Charlie Jeffery, a.a.O. (2003), S. 81.

25 Kerstin Völkl und Kai-Uwe Schnapp stellen in ihren Analysen ebenfalls einen starken Einfluss der Landespolitik bei einer gleichzeitig relativ schwachen Wirksamkeit bundespolitischer Faktoren auf das Abstimmungsverhalten bei den Landtagswahlen fest. Vgl. hierzu Kerstin Völkl, Welchen Einfluss hat die Bundespolitik auf die Wahlentscheidung der Bürger bei Landtagswahlen? Eine Analyse von Individualdaten im Bundesländer- und Zeitvergleich, in: ZParl, 38. Jg. (2007), H.3, S. 480 - 491; Kai-Uwe Schnapp, Landtagswahlen und Bundespolitik: immer noch eine offene Frage? Neue Antworten im Ländervergleich auf Aggregatdatenbasis, in: ebenda, S. 463 - 480.

26 Daniel Hough / Charlie Jeffery, a.a.O. (2003), betrachteten die Entwicklung der relativen Stimmenanteile der Parteien über die Zeit. Dagegen analysierten sie individuelles Wahlverhalten nicht direkt. Eine Mikrofundierung ihrer impliziten Annahmen mit Individualdaten ist aber durchaus aufschlussreich, wenn es darum geht, Aussagen über individuelle Wählermotive noch verlässlicher zu machen. Zur Debatte um die Problematik von ökologischen Schlüssen vgl. David A. Freedman / Stephen P. Klein / Michael Ostland / Michael R. Roberts, A Solution to the Ecological Inference Problem, in: Journal of the American Statistical Association, 93. Jg. (1998), S. 1518 - 1522; Gary King, The Future of Ecological Inference Research: A Comment on Freedman et al., in: Journal of the American Statistical Association, 94. Jg. (1999), S. 352 - 357 (mit Diskussion). 


\subsection{Deskriptive Befunde}

Auf Nachfrage antworteten im Durchschnitt 78,6 Prozent der Befragten in Westdeutschland, dass die Bundespolitik eine wichtige Rolle bei ihren Wahlerwägungen spiele. ${ }^{27} \mathrm{Im}$ Vergleich dazu liegt der mittlere Anteilswert in den neuen Bundesländern bei 87,2 Prozent. Dies lässt auf eine hohe Bedeutung der übergeordneten bundespolitischen Konstellation in beiden Teilen der Bundesrepublik schließen, wobei in Ostdeutschland mit einer Differenz von beinahe neun Prozentpunkten im Vergleich zu den alten Bundesländern die Bundespolitik wichtiger zu sein scheint (vgl. Tabelle 1).

\begin{tabular}{|l|c|c|c|c|c|c|c|c|}
\hline Tabelle 1: Wichtigkeit der Bundespolitik bei Landtagswablentscheidungen, 1997 bis 1999 \\
(Angaben in Prozent)
\end{tabular}

Anteilswerte von Landtagswahlstudien, bei denen die folgende Frage gestellt wurde (Fragevariante Nummer 1): „Wenn Sie an Ihre Wahlentscheidung für die Landtagswahl in XXX denken, wie wichtig ist da für Sie die Bundespolitik?"

Quelle: Eigene Zusammenstellung auf Basis der Landtagswahlstudien der Forschungsgruppe Wahlen (siehe Anhang).

\begin{tabular}{|l|l|l|l|l|l|l|l|l|l|l|}
\hline Tabelle 2: Relative Wichtigkeit von Bundespolitik versus Landespolitik bei Landtagswahl- \\
entscheidungen, $1999 / 2000$ (Angaben in Prozent) \\
\hline \\
\hline
\end{tabular}

27 Die drei Landtagswahlstudien Baden-Württemberg 1996, Rheinland-Pfalz 1996 und SchleswigHolstein 1996 werden in diesem deskriptiven Abschnitt nicht berücksichtigt, da die hier analysierten Items nicht abgefragt wurden. 
In späteren Umfragen, beginnend mit der Landtagswahlstudie in Bremen 1999, veränderte die Forschungsgruppe Wahlen die Fragestellung und formulierte die Indikatoren neu. Die Wichtigkeit der Bundespolitik wurde nun direkt in Relation zur Wichtigkeit der landespolitischen Verhältnisse abgefragt (vgl. Tabelle 2).

Führt man die Landespolitik als einen expliziten Vergleichsanker ein, dann geben im Westen durchschnittlich nur 33,1 Prozent der Befragten an, dass die Bundespolitik bei ihrer Abstimmungsentscheidung eine wichtigere Rolle spielt als die Landespolitik. Im Verhältnis dazu steigt dieser mittlere Anteilswert im Osten um über acht Punkte auf 41,4 Prozent an. Bei einem direkten Vergleich der Bundes- mit der Landesebene beeinflusst also die landespolitische Dimension in beiden Teilen Deutschlands die individuellen Landtagswahlpräferenzen stärker. Dabei spielt jedoch die Bundespolitik bei den ostdeutschen Wählern eine wichtigere Rolle als in den alten Bundesländern. Die bundespolitische Durchdringung der Landtagswahlen ist damit in den neuen Bundesländern stärker als im Westen. Dieser Befund steht mit den Anteilswerten aus Tabelle 1 in Einklang.

\subsection{Operationalisierung der Forschungsvariablen}

Um die Regionalisierungsthese einer empirischen Überprüfung mit multiplen Regressionsmodellen zugänglich zu machen, wurden die theoretischen Konzepte folgendermaßen operationalisiert:

Landtagswahlpräferenzen: Die dichotome abhängige Variable LTWSPD(i) („Parteiwahl des i-ten Individuums: SPD versus andere Parteien“) wurde auf der Basis des Items zur Parteiwahl gebildet und weist die folgende Codierung auf: 1 = Befragter gibt an, bei der anstehenden Landtagswahl die SPD wählen zu wollen beziehungsweise hat bereits für die SPD per Briefwahl gestimmt; 0 = Befragter gibt an, bei der anstehenden Landtagswahl eine andere Partei als die SPD wählen zu wollen beziehungsweise hat bereits für eine andere Partei als die SPD per Briefwahl gestimmt. Personen, bei denen hierzu keine Angaben vorliegen, wurden aus der weiteren Analyse ausgeschlossen. Analog hierzu wurde auch die andere abhängige Variable LTWCDU(i) („Parteiwahl des i-ten Individuums: CDU versus andere Parteien“) gebildet: 1 = Befragter gibt an, bei der anstehenden Landtagswahl die CDU wählen zu wollen beziehungsweise hat bereits für die CDU per Briefwahl gestimmt; $0=$ Befragter gibt an, bei der anstehenden Landtagswahl eine andere Partei als die CDU wählen zu wollen beziehungsweise hat bereits für eine andere Partei als die CDU per Briefwahl gestimmt.

Beurteilungen der Landesparteien: Diese wurden im Rahmen der Telefonumfragen durch speziell dafür konstruierte Sympathieskalen erfasst. Diese "Skalometer“ bilden in den zu schätzenden Modellen die regionale Dimension einer Landtagswahl ab. Dabei geht der Wertebereich jeweils von 1 („Befragter hält überhaupt nichts von der Partei“) bis 11 („Befragter hält sehr viel von der Partei“). Mit Werten zwischen diesen beiden Extremen konnten die Befragten ihre Sympathiebekundung abgestuft angeben. Die entsprechenden unabhängigen Variablen lauten SPDLA(i) („Sympathieskalometer Landes-SPD für den Befragten i“) und CDULA(i) („Sympathieskalometer Landes-CDU für den Befragten i“).

Beurteilung der Bundesparteien: Hierfür wurden identisch konstruierte Skalen für die subjektiven Sympathiewerte verwendet. Die Bezeichnungen der dazugehörigen unabhängigen Variablen lauten: SPDBU(i) („Sympathieskalometer Bundes-SPD für den Befragten i“) und CDUBU(i) („Sympathieskalometer Bundes-CDU für den Befragten i“). 
Es wurden ausschließlich Analysen für die beiden größten Parteien im bundesdeutschen Regierungssystem, CDU und SPD, durchgeführt. Auf eine Hinzunahme kleinerer Parteien (FDP, Grüne und PDS) wurde verzichtet, da mit der analysierten Datenbasis ein direkter West-Ost-Vergleich über weite Strecken nicht möglich ist: Die Sympathiewerte für die PDS wurden nur in den neuen Bundesländern erhoben, während im Falle der FDP und der Grünen die benötigten Informationen in der Regel nur für die alten Bundesländer vorliegen.

\section{4. „Replikativer Survey“ als Forschungsdesign}

Die der Analyse zugrunde liegende Forschungsanordnung ist ein „replikativer Survey“28. Bei den Landtagswahlstudien der Forschungsgruppe Wahlen handelt es sich um Querschnittserhebungen, bei denen wiederholt zu verschiedenen Zeitpunkten anhand weitgehend identischer Fragebögen Personen aus jeweils unterschiedlichen Stichproben telefonisch interviewt wurden. Diese Ausgangssituation eignet sich hervorragend dazu, ein und dasselbe statistische Modell in vielen verschiedenen Datensätzen zu replizieren und dadurch seine Robustheit zu testen. Die abhängigen Variablen stellen jeweils ein dichotomes Merkmal mit nur zwei möglichen Ausprägungen dar. Solche binären Variablen lassen sich besonders adäquat mit logistischen Regressionsmodellen analysieren. ${ }^{29}$ Zunächst wurden in jedem der 17 Datensätze die folgenden beiden logistischen Regressionsmodelle geschätzt:

1) $\ln ($ W[LTWSPD $(i)=1]: \operatorname{W}[\operatorname{LTWSPD}(i)=0])=a+b 1 \cdot \operatorname{SPDBU}(\mathrm{i})+\mathrm{b} 2 \cdot \operatorname{SPDLA}(\mathrm{i})$

2) $\ln (\mathrm{W}[\operatorname{LTWCDU}(\mathrm{i})=1]: \mathrm{W}[\operatorname{LTWCDU}(\mathrm{i})=0])=\mathrm{a}+\mathrm{b} 1 \cdot \operatorname{CDUBU}(\mathrm{i})+\mathrm{b} 2 \cdot \operatorname{CDULA}(\mathrm{i})$

wobei:

LTWSPD $(\mathrm{i})=$ Parteiwahl bei den Landtagswahlen für das i-te Individuum

$(1=\mathrm{SPD} ; 0=$ andere Partei $)$.

LTWCDU(i) = Parteiwahl bei den Landtagswahlen für das i-te Individuum

$(1=\mathrm{CDU} ; 0$ = andere Partei).

SPDBU(i) = Sympathie für Bundes-SPD beim i-ten Individuum

(Skalometer: 1 = „vollständig unzufrieden“ bis $11=$,voll und ganz zufrieden“).

SPDLA(i) = Sympathie für Landes-SPD beim i-ten Individuum.

CDUBU(i) = Sympathie für Bundes-CDU beim i-ten Individuum .

CDULA(i) = Sympathie für Landes-CDU beim i-ten Individuum.

„W“ bedeutet in diesem Zusammenhang „Wahrscheinlichkeit“; mit „ln“ ist der natürliche

Logarithmus gemeint.

28 Glenn Firebaugh, Analyzing Repeated Surveys, Thousand Oaks 1997. Replikative Surveys sind genaue Nachbildungen früher durchgeführter Umfragen. Hierbei werden Variablen zu verschiedenen Zeitpunkten durch dieselbe Operationalisierung gemessen. Diese Messung wird aber im Gegensatz zu einem Panel-Design jeweils bei unterschiedlichen Personen durchgeführt.

29 Logistische Regressionsmodelle (synonym: „Logit-Modelle“) sind nicht-lineare Wahrscheinlichkeitsmodelle, anhand derer die Einflussstärken der Landes- und der Bundesparteien auf die individuellen Wahlpräferenzen geschätzt und verglichen wurden. Vereinfacht dargestellt lassen sich mit den geschätzten „Chancenkoeffizienten“ für beliebige Kombinationen der Sympathiewerte von Landes- und Bundesparteien die Chancen berechnen, bei der nächsten Landtagswahl für die jeweils betrachtete Partei (CDU oder SPD) zu stimmen. Für eine verständliche Erläuterung der Basiskonzepte von logistischen Regressionen sei verwiesen auf Scott Menard, Applied Logistic Regression Analysis, 2. Auflage, Thousand Oaks 2002. 
Bei der Interpretation der geschätzten Regressionskoeffizienten sind vor allem die Betrachtung der Vorzeichen sowie ein Vergleich der relativen Größe der Koeffizienten zueinander relevant. Beispielsweise bedeutet ein positives Vorzeichen des Regressionskoeffizienten von SPDLA(i), dass die Wahrscheinlichkeit, bei der Landtagswahl die SPD zu wählen, mit zunehmender Sympathie für die Landes-SPD ansteigt; ein negatives Vorzeichen würde dagegen bedeuten, dass die entsprechende Wahrscheinlichkeit sinkt. Dabei ist das Ausmaß der Veränderung umso stärker, je größer der Betrag des Koeffizienten ausfällt. ${ }^{30}$ Als eine beobachtbare Implikation der Regionalisierungshypothese wird erwartet, dass die Bundespartei bei der individuellen Landtagswahlentscheidung eine schwächere Rolle spielt als die Landespartei, was sich auch in den relativen Größen der jeweiligen Koeffizienten niederschlagen sollte. Es wäre demnach zu erwarten, dass in den Modellen die Regressionskoeffizienten der Landesparteien signifikant größer sind als die Regressionskoeffizienten der Bundesparteien. Formal kann die theoretische Erwartung der Regionalisierungsthese dargestellt werden als:

\section{SPDLA(i) > SPDBU(i) bzw. CDULA(i) > CDUBU(i).}

Um diese Annahme empirisch zu überprüfen, wurde jeweils in allen Regressionsmodellen ein Signifikanztest („Wald-Test“) auf Gleichheit der entsprechenden Regressionskoeffizienten durchgeführt (Nullhypothese: $\operatorname{SPDLA}(\mathrm{i})=\operatorname{SPDBU}(\mathrm{i})$ beziehungsweise CDULA(i) = CDUBU(i)).

\subsection{Empirische Befunde}

Die beiden oben beschriebenen Modellgleichungen wurden auf identische Weise in allen 17 Datensätzen geschätzt. Insgesamt wurden 34 logistische Regressionen berechnet. Zusätzlich ist im Anhang eine intuitiv zugänglichere graphische Interpretationshilfe der empirischen Befunde aufbereitet (vgl. hierzu Abbildung 1). Die Tabellen 3 bis 6 bilden die Ergebnisse der Berechnungen getrennt nach West und Ost ab. Die Werte in den Tabellen 3 und 5 wurden auf Grundlage der in Abschnitt 3.4. präsentierten Gleichung 1 für die SPDWahl, die Werte für die CDU-Wahl in den Tabellen 4 und 6 wurden mit Gleichung 2 geschätzt. Im ersten Schritt wurden für jede Landtagswahlstudie die Regressionskoeffizienten b1 und b2 (rechte Seite der Gleichungen) geschätzt, im zweiten Schritt wurden die geschätzten Regressionskoeffizienten durch einfaches Exponieren in Chancenverhältnisse (so genannte Odds-Ratios) umgerechnet. Allgemein gilt für Effektstärken („Odds Ratios“) größer 1: Der Effekt ist umso stärker, je höher der Betrag der geschätzten Effekte ausfällt. Die zugehörigen Werte sind in den Tabellen unter „Effektstärken“ abgetragen (ein Ablesebeispiel findet sich in Tabelle 5).

Bei allen 34 Replikationen der logistischen Regressionen kann ein akzeptabler bis guter Gesamt-Fit des Modells festgestellt werden. ${ }^{31}$ Zudem deuten alle LR-Chi2-Werte auf einen

30 Vgl. Ulrich Kohler / Frauke Kreuter, Datenanalyse mit Stata, München und Wien 2001.

31 Als Gütemaß für die Beurteilung der Modelle wurde jeweils das Pseudo R2 nach McFadden herangezogen. Bei der Berechnung von McFadden-Pseudo-R2 wird jeweils der Quotient der Likelihood des gegebenen Modells zur Likelihood eines Modells, bei dem alle Parameter auf null ge- 
signifikanten Modellfit hin ( $\mathrm{p} \leq 0.01$ ), und in keinem einzigen Fall liegt eine Modellgefährdung durch eventuelle Multikollinearitätsprobleme vor. Alle berechneten Varianzinflationsfaktoren (VIF) sind kleiner als der kritische Wert 5. Nähere Erläuterungen zur Multikollinearitätsprüfung sind dem Anhang zu entnehmen.

In den Landtagswahlstudien für die alten Bundesländer (vgl. Tabellen 3 und 4) stützen die empirischen Befunde die Regionalisierungsthese: Bei 15 von insgesamt 22 Modellen finden sich die Vermutungen von Daniel Hough und Charlie Jeffery bestätigt. Demnach haben die Landesparteien, relativ zu den Bundesparteien, stärkeren Einfluss auf die individuellen Abstimmungswahrscheinlichkeiten bei den Landtagswahlen. Anders formuliert: In 15 von insgesamt 22 berechneten logistischen Regressionen für die alten Bundesländer ist der jeweilige Koeffizient für die Landespartei (SPDLA(i) beziehungsweise CDULA(i)) auf einem der drei konventionellen Niveaus signifikant größer als der entsprechende Koeffizient für die Bundespartei (SPDBU(i) beziehungsweise CDUBU(i)). Dies entspricht den eingangs formulierten theoretischen Erwartungen der Regionalisierungsthese. Dagegen kann bei den übrigen sieben Modellen für Westdeutschland keine signifikante Differenz der beiden Koeffizienten anhand des Wald-Tests festgestellt werden. Somit kann für die alten Bundesländer im betrachteten Analysezeitraum die These vom „Mehr-EbenenWahlverhalten " tendenziell bestätigt werden. ${ }^{32}$ Die Wähler im Westen scheinen demnach hinreichend zwischen Landespolitik und Bundespolitik zu differenzieren, wobei hier landesparteipolitische Aspekte die individuelle Landtagswahlpräferenz zumeist stärker determinieren als bundesparteipolitische Sympathiepunkte.

Die empirischen Befunde für Ostdeutschland liefern dagegen ein anderes Bild (vgl. Tabellen 5 und 6): Elf der insgesamt zwölf für die neuen Bundesländer durchgeführten WaldTests stützen nicht die Regionalisierungshypothese. In zehn Fällen kann die Nullhypothese auf Gleichheit der beiden Regressionskoeffizienten (SPDLA(i) = SPDBU(i) beziehungsweise CDULA(i) = CDUBU(i)) nicht verworfen werden. In einem Fall ist sogar ein signifikant stärkerer Einfluss $(\mathrm{p} \leq 0.05)$ der Sympathiewerte der Bundespartei auf die individuelle Wahlpräferenz erkennbar (vgl. Tabelle 6: CDU-Landtagswahl in Sachsen-Anhalt 1998). Nur in einem einzigen Fall stützen die Daten für Ostdeutschland die Regionalisierungsthese auf einem empirischen Signifikanzniveau von einem Prozent (vgl. Tabelle 5: SPD-Landtagswahl in Brandenburg 1999). Somit kann für die neuen Bundesländer im untersuchten Zeitraum festgehalten werden, dass die individuellen Landtagswahlpräferenzen von den Bundesparteien ähnlich stark beeinflusst werden wie von den Landesparteien. Das bundesparteipolitische Geschehen scheint damit im Osten einen relativ stärkeren Einfluss auf die Landtagswahlpräferenzen auszuüben als im Westen. Die empirischen Befunde für Ostdeutschland liefern somit wenig Evidenz für die Regionalisierungsthese. Dieses Ergebnis wird auch durch die oben diskutierten deskriptiven Befunde (siehe Tabellen 1 und 2) untermauert.

Als Ursache für die starke Verflechtung von Bundes- und Landesparteipolitik im Bewusstsein der ostdeutschen Wähler lässt sich die seit der Wende noch nicht abgeschlossene

setzt sind, gebildet: Pseudo R2 = $1-\ln \left(\mathrm{L}_{1}\right): \ln \left(\mathrm{L}_{0}\right)$; vgl. David W. Jr. Hosmer / Stanley Lemeshow, Applied Logistic Regression, New York 1989, S. 149. Hierbei wurden Werte zwischen 0.207 (vgl. Tabelle 5: SPD-Landtagswahl in Mecklenburg-Vorpommern 1998) und 0.438 (vgl. Tabelle 3: SPD-Landtagswahl in Saarland 1999) erreicht.

32 Daniel Hough / Charlie Jeffery, a.a.O. (2003), S. 81. 


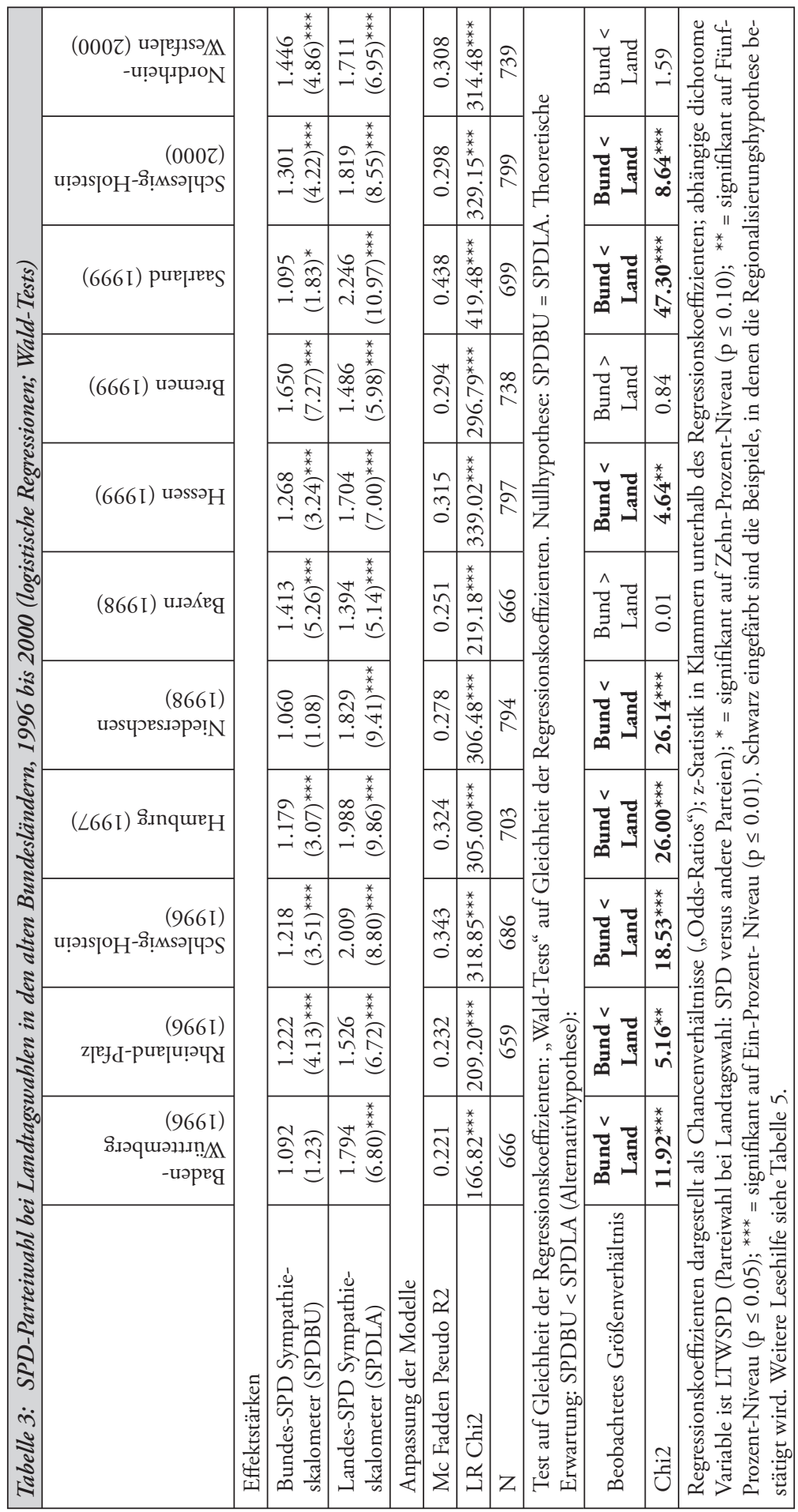




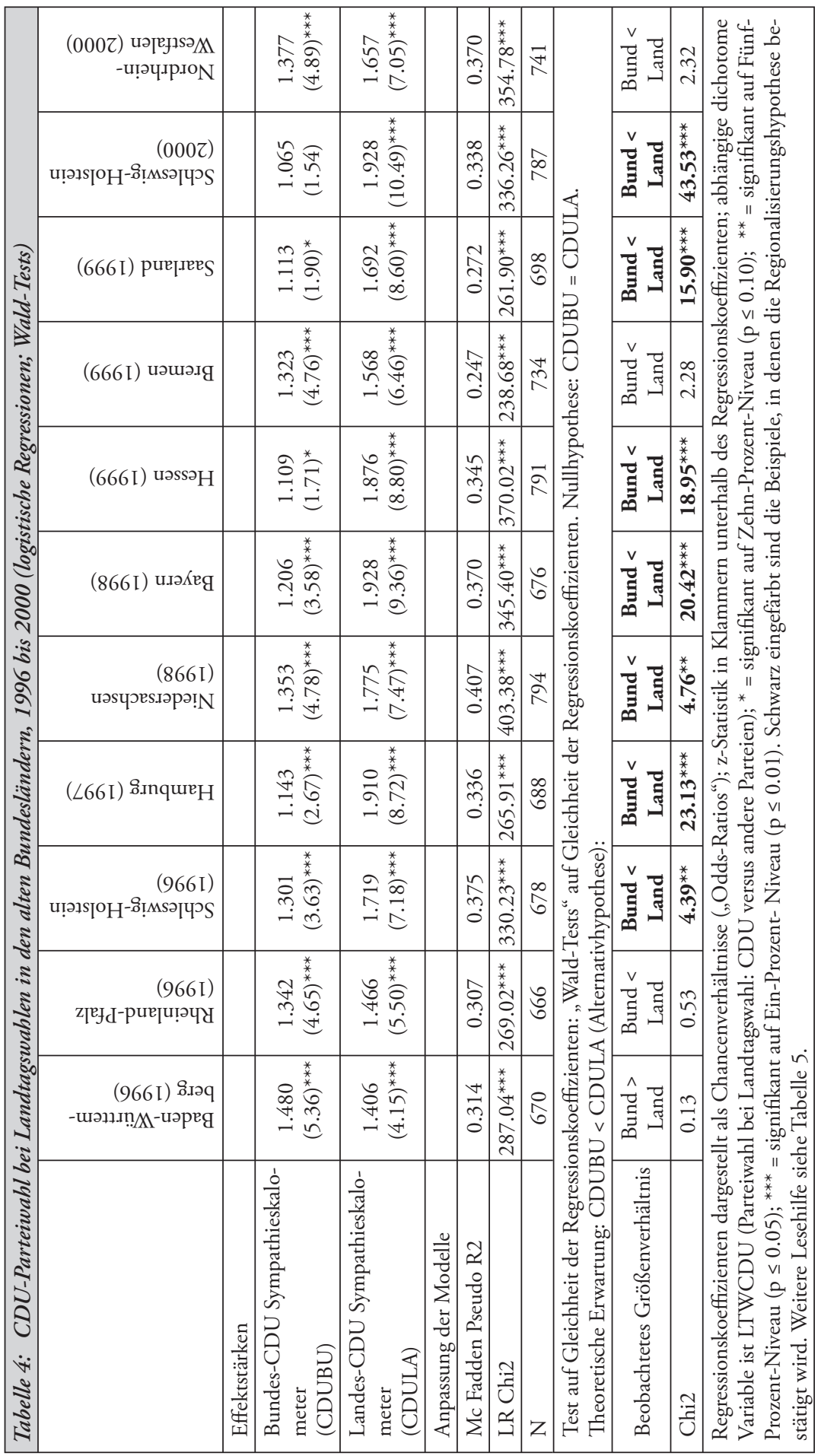


Konsolidierung des bundesdeutschen Parteiensystems in Ostdeutschland anführen. Darauf deuten vor allem die generell schwächeren Parteibindungen und die höhere Wechselwahlbereitschaft in den neuen Bundesländern hin. ${ }^{33}$ Stärker als im Westen wählen Bürger im Osten eher situationsbezogen, wobei Orientierungen an einzelnen Spitzenpolitikern sowie aktuellen Themen vorherrschend sind. ${ }^{34}$ Folglich liegt die Vermutung nahe, dass das bundespolitische Tagesgeschehen bei den ostdeutschen Landtagswahlen einen weitaus stärkeren Einfluss ausübt als in Westdeutschland. Empirische Unterstützung für diese Annahme lässt sich in der quantitativen Studie von Simone Burkhart finden: „Gerade in den neuen Bundesländern ist der Einfluss der Bundespolitik auf die Wahlentscheidung bei Landtagswahlen besonders hoch. " 35 Im Gegensatz dazu können sich die Wähler im Westen auf ungleich längere Erfahrungen mit dem bestehenden Parteiensystem stützen. So haben sich im Laufe der Jahrzehnte in den alten Bundesländern regionale Hochburgen der beiden großen Volksparteien CDU und SPD mit relativ stabilen lokalen Verwurzelungen und eigenen Landesprofilen herausgebildet. ${ }^{36}$ Diese traditionell stark ausdifferenzierte Mehrebenenstruktur des bundesdeutschen Parteiensystems hat sich offenbar im Lauf der Zeit im Bewusstsein der westdeutschen Wähler verankert.

Als weiterer Grund für die relativ starke bundespolitische Durchdringung der ostdeutschen Landtagswahlen kann der nur schleppend vorankommende „Aufbau Ost“ gesehen werden. Vor allem die nach wie vor hohe Arbeitslosigkeit in den neuen Bundesländern hat sich zu einem bundespolitischen Dauerthema entwickelt und heizt die Proteststimmung in der ostdeutschen Bevölkerung weiter an. Das Protest- beziehungsweise Sanktionswahlverhalten gegen die jeweils amtierende Bundesregierung im Rahmen von Landtagswahlen ist im Osten viel stärker verbreitet als im Westen. Hierauf deutet auch eine zusätzliche Analyse der amtlichen Wahlstatistik für den Zeitraum von 1990 bis 2002 hin: So büßten die jeweils amtierenden Bundesregierungsparteien bei den westdeutschen Landtagswahlen relativ zu ihren Ergebnissen bei den Bundestagswahlen in einem gegebenen Bundesland im Durchschnitt 9,5 Prozentpunkte ihrer erwarteten Stimmen ein. Demgegenüber fiel bei den Landtagswahlen in Ostdeutschland mit einem im Vergleich zu den Bundestagswahlen mittleren relativen Stimmeneinbruch von 17,3 Prozentpunkten die „Bestrafung“ der Bundesregierungsparteien wesentlich härter aus. ${ }^{37}$ Dieser Befund stützt die Vermutung, dass das Protest- beziehungsweise Sanktionswahlverhalten gegen die jeweils amtierende Bundesregierung bei Landtagswahlen in den neuen Bundesländern viel stärker verbreitet ist als in Westdeutschland. Auch die neueren Ergebnisse von Armin Schäfer, die auf eine nach wie vor stärker ausgeprägte Sozialstaatsorientierung der ostdeutschen Wähler hindeuten, stüt-

33 Vgl. Cornelia Weins, The East German Vote in the 1998 General Election, in: German Politics, 8. Jg. (1999), S. $48-71$.

34 Vgl. Kai Arzheimer / Jürgen W. Falter, Ist der Osten wirklich rot? Das Wahlverhalten bei der Bundestagswahl 2002 in Ost-West-Perspektive, in: APuZ, B 49 - 50 (2002), S. 27 - 35.

35 Vgl. Simone Burkhart, a.a.O. (2005), S. 24.

36 Man denke in diesem Zusammenhang etwa nur an die CSU in Bayern mit ihrer bewussten Abgrenzung zur Bundes-CDU.

37 Die Berechnungen der relativen Stimmenverluste der Bundesregierungsparteien bei Landtagswahlen folgen dem von Dinkel vorgeschlagenen Verfahren; vgl. Reiner Dinkel, a.a.O. (1977). Hierbei werden in einem gegebenen Bundesland die Wahlergebnisse der jeweils amtierenden Bundesregierungsparteien bei Landtagswahlen in Relation gesetzt zu ihren Stimmenanteilen bei den Bundestagswahlen. 


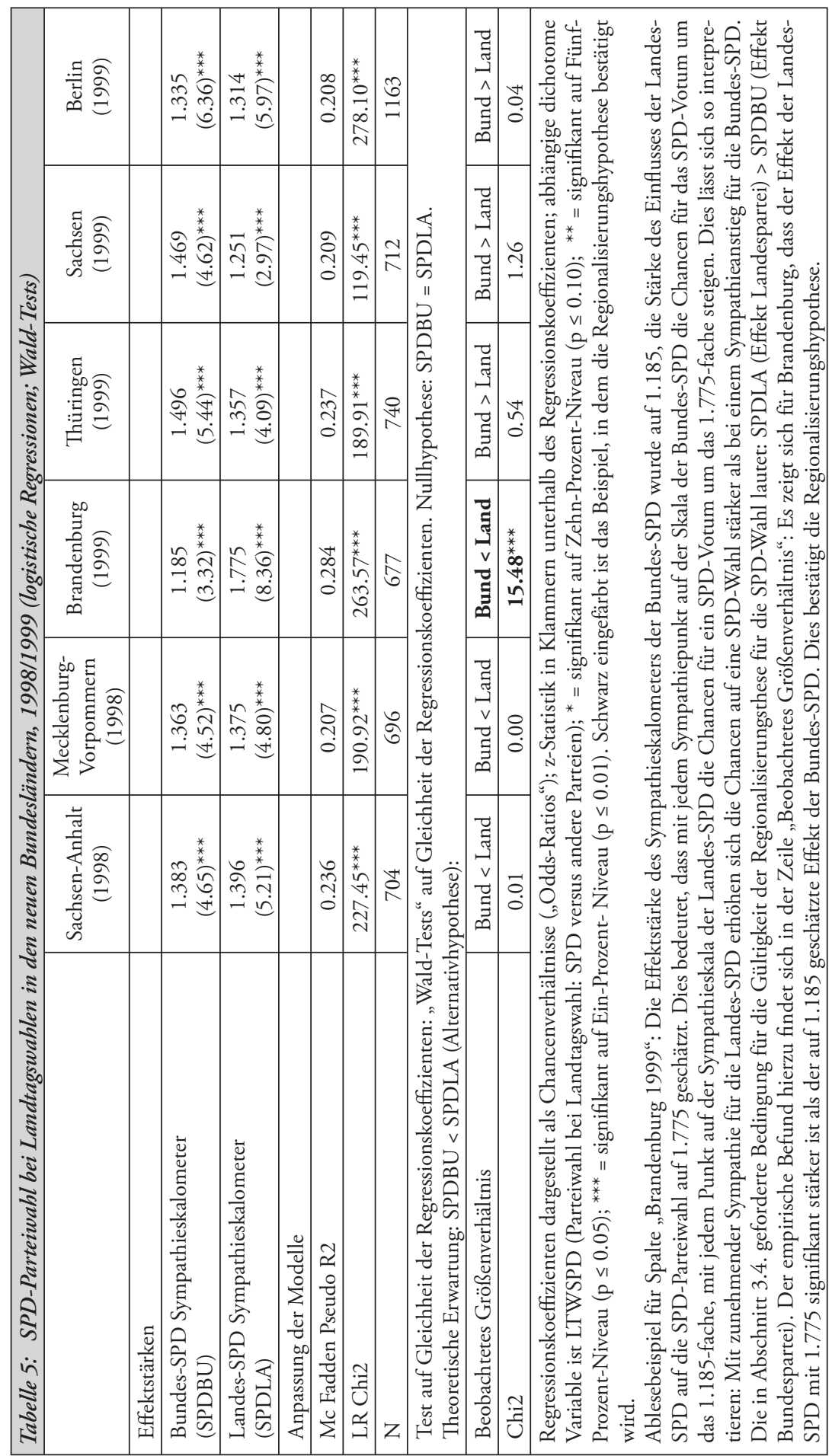




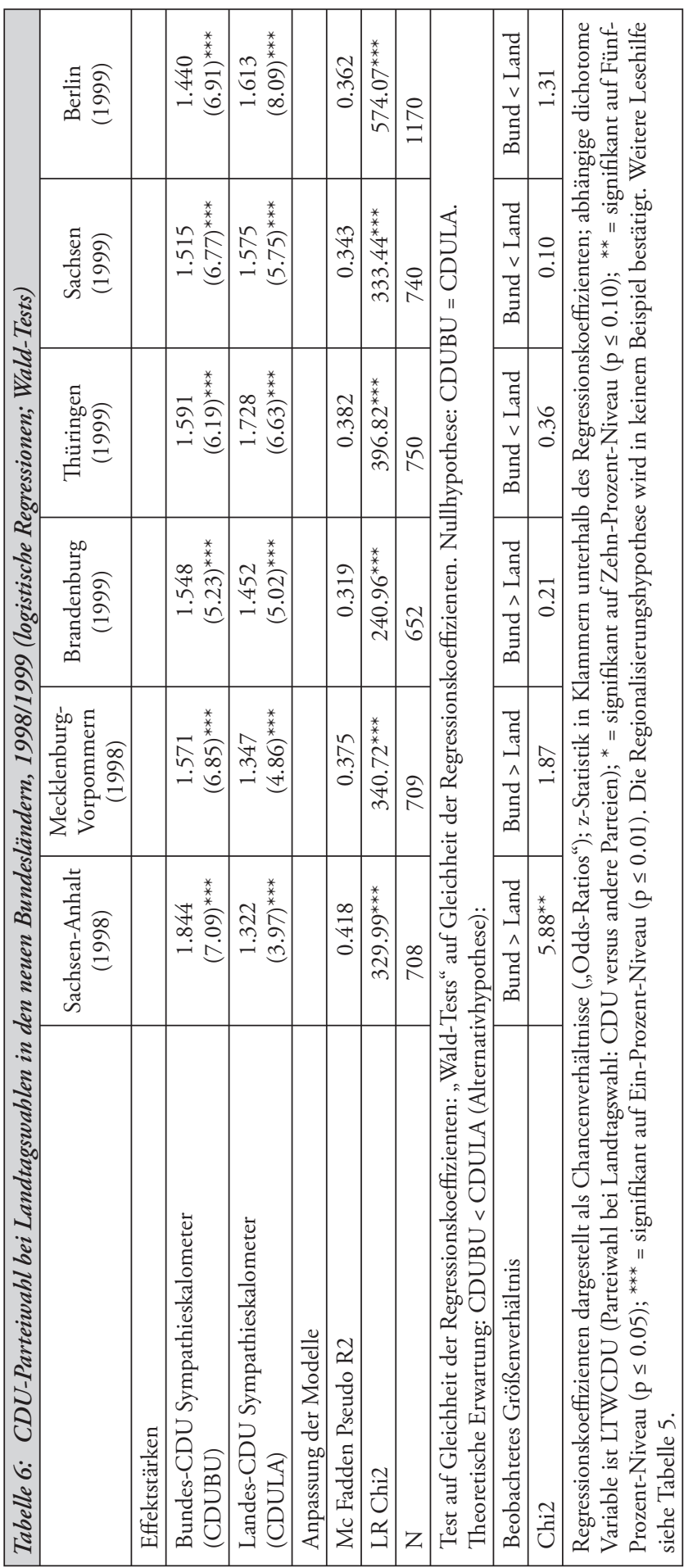


zen diese Annahme. Demnach ist bei tiefen Einschnitten in das soziale Netz in Ostdeutschland mit größerem Widerstand zu rechnen. ${ }^{38}$

In der vorliegenden quantitativen Wahlanalyse wurden abstrahiert vom Einzelfall einer konkreten Landtagswahl ein allgemeiner Analyserahmen präsentiert und einige generelle Tendenzen bezüglich des Abstimmungsverhaltens bei Landtagswahlen herausgearbeitet. Grundsätzlich besteht bei generalisierenden empirischen Studien immer die Gefahr der Nichtberücksichtigung wichtiger Einflussfaktoren in den statistischen Modellen. So konnten in den Regressionsmodellen etwa situative Faktoren und besondere Einzelereignisse ${ }^{39}$, welche die individuellen Landtagswahlpräferenzen beeinflussen können, nicht berücksichtigt werden. Trotz dieser notwendigen Vereinfachungen bietet das vorgestellte Analyseverfahren vielfältige Erweiterungsmöglichkeiten für weitere Untersuchungen. ${ }^{40}$

Generell könnte die zeitliche Positionierung einzelner Landtagswahlen die Ergebnisse der vorliegenden Studie beeinflussen: In der Mitte des Untersuchungszeitraums (1998) änderten sich die bundespolitischen Mehrheitsverhältnisse. So verlor bei der Bundestagswahl am 27. September 1998 die damals amtierende schwarz-gelbe Bundesregierung unter Helmut Kohl ihre Mehrheit im Bundestag. Hierbei ist zu vermuten, dass der im Vorfeld zur Bundestagswahl medienwirksam inszenierte Wahlkampf nicht ohne Einfluss war auf Landtagswahlen, die in zeitlicher Nähe zur Bundestagswahl lagen (plus/minus drei Monate). Dies betrifft insbesondere die Landtagswahl am 13. September 1998 in Bayern und die zeitgleich mit der Bundestagswahl abgehaltene Landtagswahl am 27. September 1998 in Mecklenburg-Vorpommern. Anhand der analysierten Daten lässt sich diese Vermutung teilweise bestätigen: In Bayern sind die Effekte von Bundes-SPD (1.413) und Landes-SPD (1.394) auf die SPD-Parteiwahl ungefähr gleich stark. Dagegen beeinflusste die bayerische CSU (1.928) die Landesparteiwahl signifikant stärker als die Bundes-CDU (1.206). Aufgrund der Sonderstellung der CSU im bundesdeutschen Parteiensystem ist dieser Befund allerdings nicht unerwartet. In Mecklenburg-Vorpommern sind die Effekte von BundesSPD (1.363) und Landes-SPD (1.375) ähnlich wie in Bayern ungefähr gleich stark. Dagegen lässt sich für die CDU-Landesparteiwahl ein im Vergleich zur Landes-CDU (1.347)

38 Armin Schäfer, Die Reform des Sozialstaats und das deutsche Parteiensystem: Abschied von den Volksparteien?, in: ZParl, 38. Jg. (2007), H. 3, S. 648 - 666.

39 Hierunter sind bundes- beziehungsweise landespolitische Geschehnisse zu subsumieren, die in der Regel unerwartet eintreten und einer Partei unverhofft großen Schaden zufügen oder einen Sympathieaufschwung bringen können. Nach der deutschen Wiedervereinigung beispielsweise musste die Bundesregierung (CDU/CSU-FDP) im Februar 1991 die Steuern erhöhen, um die hohen Kosten der Einheit finanzieren zu können. Da im Bundestagswahlkampf etwas anderes versprochen wurde, fühlten sich viele Wähler durch diese Entscheidung betrogen und nahmen kurz darauf die rheinland-pfälzischen Landtagswahlen am 21. April 1991 zum Anlass, den großen Koalitionspartner CDU durch Stimmenverweigerung beziehungsweise Alternativenwahl für die „Steuerlüge“ abzustrafen (minus 6,4 Prozentpunkte im Vergleich zur vorhergehenden Landtagswahl). Dagegen konnte die SPD unmittelbar von diesen Ereignissen profitieren und sechs Prozentpunkte dazugewinnen. Vgl. hierzu Frank Decker / Julia von Blumenthal, a.a.O. (2001), S. 150. Als landespolitisches Beispiel kann hier die Barschel-Affäre in Schleswig-Holstein aufgeführt werden, die der SPD bei der Landtagswahl am 8. Mai 1988 starke Stimmenzugewinne bescherte und gleichzeitig bei der CDU zu einem massiven Einbruch in den Wählerstimmen führte. Vgl. hierzu ebenda, S. 147.

40 So haben $O h r$ und Klein in ihrer Analyse der nordrhein-westfälischen Landtagswahlen die hier präsentierten Modelle erweitert und zusätzlich die Kandidatenbewertungen für das Amt des Ministerpräsidenten in ihr Modell aufgenommen. Vgl. Dieter Ohr / Markus Klein, a.a.O. 
stärkerer Einfluss der Bundes-CDU (1.571) beobachten. Jedoch ist der festgestellte Unterschied nicht signifikant. Insgesamt lässt sich somit im Bundestagswahlmonat September 1998 ein relativ starker Einfluss der Bundespolitik auf Landtagswahlen beobachten (bei drei von vier geschätzten Modellen).

\section{Diskussion und Ausblick}

In der laufenden Kontroverse, ob das Abstimmungsverhalten bei Landtagswahlen zunehmend unabhängiger von der Bundespolitik wird oder ob das Bundesgeschehen nach wie vor einen beherrschenden Einfluss auf das Votum bei Landtagswahlen ausübt, zeichnet sich insbesondere in Ostdeutschland keine eindeutige Dominanz einer der beiden Ebenen ab (in zehn von zwölf geschätzten Modellen). Auch in Westdeutschland ist bei einigen Landtagswahlen ein gleich starker Einfluss von Bundes- und Landesparteien erkennbar (in sieben von 22 geschätzten Modellen). Insgesamt lässt sich bei genau der Hälfte der 34 geschätzten Regressionsmodelle keine eindeutige Dominanz einer der beiden politischen Systemebenen feststellen. Dieser Befund stützt die zwischen den beiden kontroversen Positionen vermittelnde Einschätzung von Oscar W. Gabriel und Everhard Holtmann, die insgesamt eine Tendenz zum „gesamtsystemaren“ Abstimmungsverhalten bei den Landtagswahlen konstatieren. ${ }^{41}$ Demnach bliebe auf der politischen Systemebene auch nach der jüngsten Föderalismusreform das hohe Maß an Verflechtung und Verantwortungsdiffusion weiterhin bestehen. Dies wiederum würde auf der psychologischen Mikroebene eine eindeutige ebenenspezifische $\mathrm{Zu}$ ordnung von Wählermotiven nur schwer, allenfalls konditional möglich machen.

Für zukünftige Forschungsprojekte können die bisherigen Modelle theoretisch erweitert werden. So fordert etwa Thomas Gschwend, Bedingungen aus einer allgemeinen Theorie abzuleiten, unter denen verstärkt nach bundespolitischen Gesichtspunkten gewählt wird. ${ }^{42}$ Hierzu liefern die neuesten empirischen Landtagswahlstudien einige Anhaltspunkte: Bundespolitische Faktoren wirken beispielsweise besonders stark, wenn Bundes- und Landtagswahl auf denselben Tag fallen ${ }^{43}$, die Wähler eine nur schwache Parteibindung zeigen ${ }^{44}$ oder dieselben Parteien die Landes- und Bundesregierung stellen. Bezogen auf den letzten Punkt stellte Gschwend bei seiner Untersuchung der Wahlen zum Berliner Abgeordnetenhaus die „Eindeutigkeit der politischen Verantwortungszuweisung“ als moderierenden Faktor fest. Demnach zeigte sich bei parteipolitisch übereinstimmenden Regierungskonstellationen in Bund und Land - und somit bei klareren Verantwortlichkeiten - ein stärkerer Bundeseinfluss auf das Votum bei Landtagswahlen. ${ }^{45}$ Zudem könnten zukünftige Studien die bundespolitische Durchdringung der Landtagswahlpräferenzen im Zusammenhang mit den ausgelassenen kleineren Parteien analysieren. Hierbei würde eine interessante Problemstellung in

41 Oscar W. Gabriel / Everhard Holtmann, a.a.O., S. 446.

42 Thomas Gschwend, Berliner Abgeordnetenhauswahlen 1979 bis 2001: keine Testwahlen für die Bundesebene, in: ZParl, 38. Jg. (2007), H. 3, S. 531 - 540.

43 Markus Steinbrecher / Eva Wenzel, Landtagswahlen in Mecklenburg-Vorpommern 1990 bis 2002: der besondere Einfluss gleichzeitig stattfindender Bundestagswahlen, in: ZParl, 38. Jg. (2007), H. 3, S. $549-559$.

44 Tatjana Rudi, Landtagswahlen in Hessen 1991 bis 2003: bundespolitische Einflüsse vor allem bei unabhängigen Wählern, in: ZParl, 38. Jg. (2007), H. 3, S. 559 - 566.

45 Thomas Gschwend, a.a.O. 
der vergleichend-systematischen Analyse von Bedingungen liegen, unter denen die jeweils kleineren Koalitionspartner stärkeren Bundeseinflüssen ausgesetzt sind als der größere Koalitionspartner. ${ }^{46}$

Um die direkte Vergleichbarkeit der geschätzten Effektstärken zwischen verschiedenen Studien beziehungsweise Ländern zu verbessern, bietet sich die Berechnung von identisch spezifizierten Regressionsmodellen an. Dazu resümieren Gabriel und Holtmann: „Die Ergebnisse (...) lassen sich allerdings nicht in allen Fällen miteinander vergleichen, denn die Kontrollvariablen, die maßgeblich sind, um die Relevanz bundespolitischer Einflussfaktoren abzuschätzen, waren unterschiedlicher Art. " 47 Auch der vorliegenden Analyse sind einem direkten Vergleich mit früheren Studien Grenzen gesetzt, da vielfach unterschiedliche Beobachtungszeiträume beziehungsweise Modellspezifikation zugrunde gelegt wurden. Um die direkte Vergleichbarkeit komplexer statistischer Modelle über einzelne Landtagswahlen hinweg sicherzustellen, könnte eine Orientierung zukünftiger Analysen am hier vorgestellten methodischen Verfahren eines „replikativen Surveys“ hilfreich sein.

\section{Anhang}

\section{Datengrundlage}

Als Datenbasis wurden ausgewählte Landtagswahlstudien der Forschungsgruppe Wahlen e.V., Mannheim (Primärforscher) verwendet. Die entsprechenden Datensätze wurden vom Zentralarchiv (ZA) für empirische Sozialforschung an der Universität zu Köln zwecks Analysezwecken zur Verfügung gestellt. Hinsichtlich einer Replikation können die Daten jederzeit beim ZA Köln beantragt werden. Konkret handelt es sich dabei um die folgenden Landtagswahlen (mit ZA-Identifikationsnummer der jeweiligen Studie): Baden-Württemberg am 24. März 1996 (ZA2913), Rheinland-Pfalz am 24. März 1996 (ZA2914), Schleswig-Holstein am 24. März 1996 (ZA2915), Hamburg am 21. September 1997 (ZA3030), Niedersachsen am 1. März 1998 (ZA3031), Sachsen-Anhalt am 26. April 1998 (ZA3032), Bayern am 13. September 1998 (ZA3167), Mecklenburg-Vorpommern am 27. September 1998 (ZA3168), Hessen am 7. Februar 1999 (ZA3120), Bremen am 6. Juni 1999 (ZA3169), Brandenburg am 5. September 1999 (ZA3895), Saarland am 5. September 1999 (ZA3896), Thüringen am 21. September 1999 (ZA3898), Sachsen am 19. September 1999 (ZA3897), Berlin am 10. Oktober 1999 (ZA 3894), Schleswig-Holstein am 27. Februar 2000 (ZA3435), Nordrhein-Westfalen am 14. Mai 2000 (ZA3436). Eine vollständige Dokumentation der hier durchgeführten Datenanalyse kann bei den Autoren angefordert werden.

\section{Prüfung auf Multikollinearität}

Allgemein liegt ein Multikollinearitätsproblem vor, wenn bei einer multiplen Regression die unabhängigen Variablen untereinander hoch korreliert sind. Dadurch werden die Stan-

46 Forschungsmethodisch könnte dabei an die hier vorgestellten beziehungsweise die von Ohr und Klein erweiterten Modelle angeknüpft werden; vgl. Dieter Ohr und Markus Klein, a.a.O. (2007). Eine Schwierigkeit könnte allerdings sein, vergleichbare Datensätze für längere Zeiträume beziehungsweise einen regionalen Vergleich zwischen West- und Ostdeutschland zu finden (vgl. hierzu auch die Ausführungen am Ende des Abschnitts 3.3.).

47 Oscar W. Gabriel/ Everhard Holtmann, a.a.O., S. 459. 
dardfehler der Regressionskoeffizienten sehr groß und die Schätzungen unpräzise. Als Konsequenz können weder die Vorzeichen noch die Koeffizientengrößen sinnvoll interpretiert werden. Auch werden überproportional häufig nicht-signifikante Regressionskoeffizienten ausgewiesen. ${ }^{48}$ In der vorliegenden Analyse würden sich aufgrund von hohen Korrelationen der unabhängigen Variablen die einzelnen Effekte (Bundespartei versus Landespartei) auf die abhängige Variable (die individuelle Landtagswahlentscheidung) nicht mehr vernünftig voneinander trennen lassen. Ein formaler Ansatz zur Aufdeckung von Multikollinearität besteht in der Ermittlung von so genannten „Varianzinflationsfaktoren“ (VIF). Diese sind definiert $\mathrm{als}^{49}: \mathrm{VIFj}=1:\left(1-\mathrm{Rj}^{2}\right)$, wobei: $\mathrm{Rj}^{2}=$ Determinationskoeffizient für die Regression einer unabhängigen Variable $\mathrm{Xj}$ gegen alle übrigen unabhängigen Variablen des Modells. VIF lässt sich für jede einzelne der unabhängigen Variablen berechnen. Dabei sind hohe Werte von VIF als Hinweis für Multikollinearität zu werten. VIF-Werte größer als fünf gelten als problematisch. In der vorliegenden Analyse werden die problematischen Grenzwerte in keinem Fall überschritten (minimale VIF-Werte: 1.733 im Westen und $2.132 \mathrm{im}$ Osten; maximale VIF-Werte: $3.690 \mathrm{im}$ Westen und $3.311 \mathrm{im}$ Osten).

\section{Graphische Interpretationshilfen für den Vergleich der relativen Effektstärken}

In Abbildung 1 wurden die zentralen Befunde der logistischen Regressionsschätzungen (vgl. Tabellen 3 bis 6) graphisch visualisiert. Dabei wurden die geschätzten Effektstärken („OddsRatios") der Bundes- und Landesparteien (y-Achse) gegen die zeitlich aufsteigenden, aneinandergereihten Landtagswahlen (x-Achse) abgetragen. Die festgestellten Phänomene, tendenziell regionalisierte Landtagswahlen im Westen auf der einen Seite und eine stärkere bundespolitische Parteipolitikverflechtung beim Landtagswahlverhalten in den neuen Bundesländern auf der anderen Seite, sind hier deutlich zu erkennen.

Im Westen liegt für die Landes-SPD die durchschnittliche Effektstärke bei 1.77, für die Bundes-SPD beträgt sie 1.27 (berechnet über einfache Mittelwertbildung der in Tabelle 3 abgebildeten Effektstärken). Die Differenz zwischen den beiden durchschnittlichen Effektstärken liegt damit für die SPD im Westen bei 0.50 zugunsten der Landesparteien. Dagegen betragen für die neuen Bundesländer die mittleren Effektstärken 1.41 für die Landes-SPD und 1.37 für die Bundes-SPD (einfache Mittelwertbildung der Effektstärken in Tabelle 5). Die durchschnittliche Differenz liegt im Osten also lediglich bei 0.04. In den Graphiken ist die im Osten engere bundespolitische Parteipolitikverflechtung klar erkennbar.

Auch beim Landtagswahlverhalten für die CDU zeigt sich ein ähnliches Muster. In den alten Bundesländern liegt die mittlere Effektstärke für die Landes-CDU bei 1.72, dagegen beträgt sie bei der Bundes-CDU lediglich 1.26 (einfache Mittelwertbildung der Effektstärke in Tabelle 4). Somit liegen die durchschnittlichen Differenzen der relativen Effektstärken im Westen bei 0.46 zugunsten der Landesparteien. Im Gegensatz dazu übersteigt bei der Wahl der CDU im Osten der Einfluss der Bundespartei den der Landespartei: Während in den neuen Bundesländern für die Landes-CDU die mittlere Effektstärke 1.51 beträgt, zeigen die Schätzungen für die Bundes-CDU einen Durchschnittswert von 1.59 (einfache Mittelwertbildung der Effektstärken in Tabelle 6). Damit liegt die mittlere Differenz im Osten bei -0.08 zuungunsten der Landesparteien.

48 Scott Menard, a.a.O., S. 76.

49 Richard A. Berk, Regression Analysis - A Constructive Critique, Thousand Oaks 2004, S. 121. 


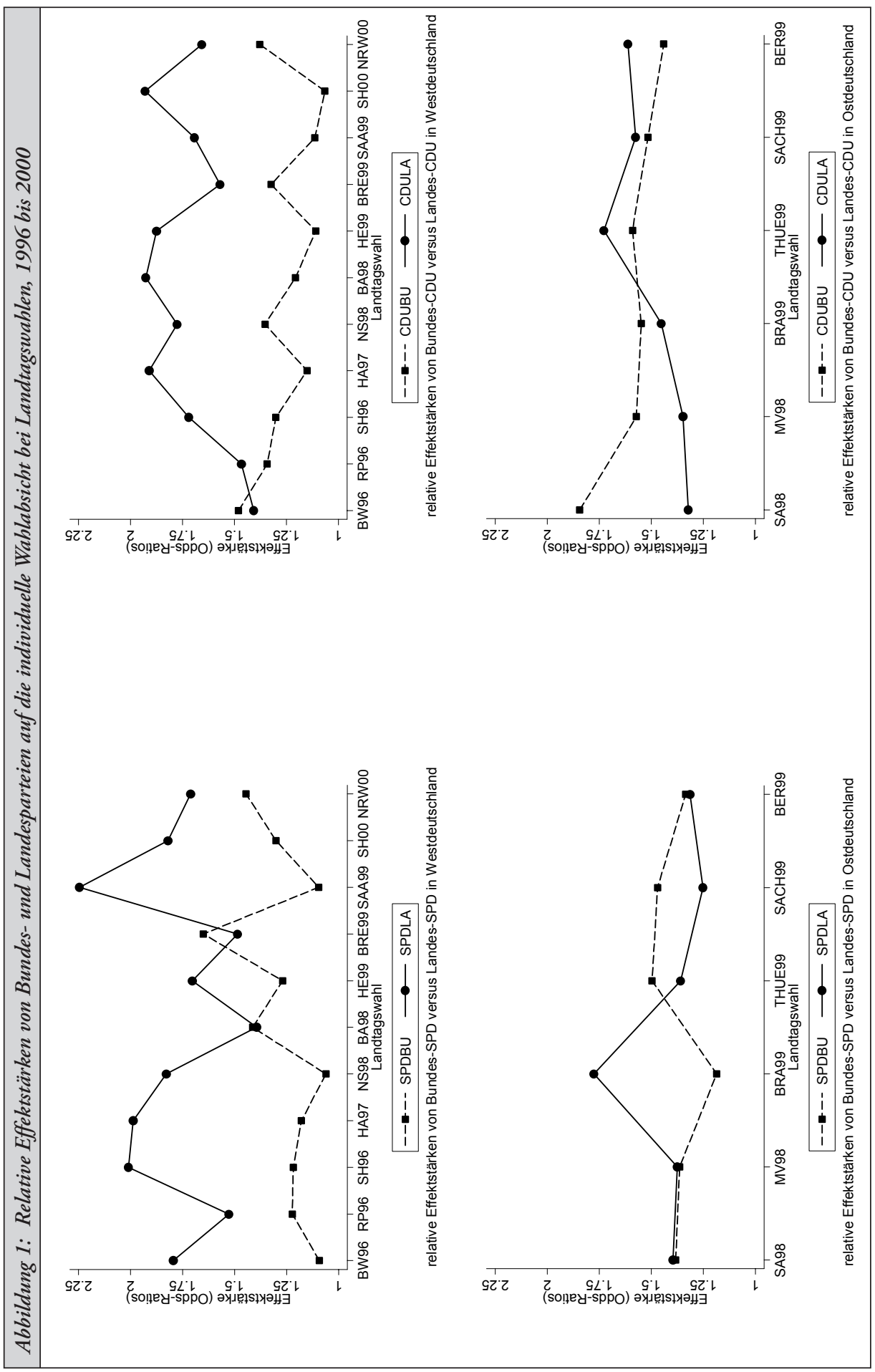

